\title{
LOS NOMBRES COMUNES EN CUANTO AL GÉNERO
}

\begin{abstract}
Resumen. El llamado «lenguaje inclusivo» trata de promover el uso generalizado de la referencia a las mujeres mediante nombres con la terminación del género femenino más habitual en español, que es la desinencia $-a$, o bien con nombres de sentido colectivo, como el profesorado, en lugar de los profesores. Se trata de evitar el masculino, pues para la ideología «inclusiva» este género solo designa varones. Sin embargo, la iniciativa tropieza con dos obstáculos que se convierten en los aspectos centrales de la controversia. Uno es el empleo del masculino genérico como término no marcado de la oposición flexiva del género y, por tanto, «inclusivo» para la referencia a ambos sexos. El otro consiste en que los nombres comunes en cuanto al género no admiten cambio de desinencia. Para sortear el primer obstáculo, se propone la coordinación del tipo los profesores y las profesoras, y para el segundo, hay quien recurre al uso de portavoza o miembra. En este trabajo se defiende que el carácter genérico del masculino es la propiedad distintiva de tres de las cinco clases de nombres que designan clases de personas, de las cuales los comunes en cuanto al género son los que han experimentado una mayor expansión en el español actual.
\end{abstract}

Palabras clave: gramática, nombre, flexión, género.

\section{Introducción}

En los estudios sobre la flexión de género en los sustantivos del español no suelen tomarse en consideración algunas cuestiones que seguramente contribuirían a aclarar la descripción. En primer lugar, desde el punto de vista metodológico, convendría distinguir entre tres tipos de nombres: los que se refieren a clases de cosas, los que indican clases de personas y los que designan clases de animales. En los tres tipos puede darse la alternancia de género masculino y femenino para un mismo lema sustantivo, pero con valores informativos muy diferentes en cada caso. En segundo lugar, en los nombres que expresan clases de personas (en adelante, nombres

\footnotetext{
* Universidad de Valladolid.
} 
personales), la relación entre la forma y el significado da lugar a cinco tipos de nombres que las gramáticas no siempre consiguen precisar. Por último, para valorar la gramaticalidad de determinados usos, interesaría conocer cuál de estos cinco tipos es el que alcanza en el español actual una mayor expansión y los factores que pueden explicar la productividad de un tipo sobre los demás.

\section{La flexión de género}

El género del sustantivo puede presentar en español un carácter léxico, muy diferente del paradigma flexivo al que pertenece (Ambadiang, 1994: $\S$ 3.2.2). No todo sustantivo femenino se interpreta como el femenino del sustantivo masculino correspondiente, pues con cierta frecuencia ambas formaciones se lematizan en el diccionario en artículos separados y diferentes, cada uno con su propia acepción. Resulta bastante productivo en esta lengua que el cambio de la desinencia de género -o / - $a$ represente además un cambio de significado, de modo que al lado de un género flexivo hay que tomar en consideración un género derivativo como el que se observa en los nombres de entidades asexuadas, pero también en nombres de profesiones, oficios, cargos, etc., referidos a clases de personas hasta hace unas décadas en el español general. Así, por un lado, con respecto a los nombres de cosas (Picallo, 2016: 633-635), la alternancia -o / - a puede expresar diferencias de tamaño o forma de ciertos objetos (barco / barca, anillo / anilla, cesto / cesta, charco / charca, huerto / huerta, bolso / bolsa, jarro / jarra, botijo / botija, río / ría, etc.), la relación entre el árbol y su fruto (manzano / manzana, almendro / almendra, avellano / avellana, guindo / guinda, cerezo / cereza, etc.), la diferencia entre contable y no contable (leño / leña), nombres de entidades individuales y colectivos (banco / banca) o diversas modificaciones del significado léxico (palabra / palabro). La proximidad con la derivación léxica de estos pares que admiten la alternancia -o / - $a$ se pone de manifiesto en el recurso a determinados sufijos derivativos para expresar relaciones semejantes a las anteriores (tomate / tomatera, higo / higuera, pera / peral, etc.). Como aportan acepciones diferentes, sería aceptable la coordinación del masculino y el femenino: Un espectáculo de lanchas, yates, barcos y barcas.

Sin embargo, los nombres de oficios y profesiones solo muy recientemente van adquiriendo el carácter gramatical propio de la morfología flexiva. Hasta el último cuarto del siglo XX, aproximadamente, no se generaliza el uso de los femeninos flexivos en sustantivos como 
notaria, médica, jueza, catedrática, portera, sacristana, tornera, priora, etc., que en los periodos anteriores representaban un cambio léxico, pues significaban, la mayoría de estos, la esposa del varón (o la religiosa de una orden) que desempeña ese cargo o profesión (Lliteras, 2008, 2010, 2014).

En la lengua actual, tras la relativa estabilidad que se ha alcanzado para designar a uno o a otro sexo mediante el cambio de desinencia flexiva (generalmente, $-o /-a$ ), sin modificación del significado léxico, la diferencia de comportamiento del género entre los nombres de cosa y los nombres personales es evidente: solo en los sustantivos que indican seres sexuados (personas y animales), la alternancia de género informa sistemáticamente sobre la identidad sexual del referente. Sin embargo, esta misma alternancia en los nombres de objetos es asistemática, pues puede hacer referencia a informaciones muy variadas, como se ha indicado. El carácter léxico del cambio de género en estos últimos nombres se revela claramente en la codificación lexicográfica, que registra cada uno de los dos lemas en artículos independientes, cada uno con su marca gramatical como masculino o femenino y con su propia definición (cesto / cesta, etc.). Además, como el género carece de carácter flexivo, no se presenta en estos pares de nombres un término no marcado que pudiera referirse genéricamente a ambos de forma indistinta (los cestos no incluye a las cestas). En cambio, el género flexivo de los nombres personales explica que las dos formas, la del masculino y la del femenino, se registren en el mismo artículo del diccionario pues comparten una misma acepción y que además, como sucede en los paradigmas flexivos, una de las formas se utilice como término no marcado.

Con respecto a los nombres de animales, también se observa una interesante diferencia entre estos y los nombres de persona. Los nombres de animales solo son de dos tipos, o bien epicenos (la pantera, la jirafa, el puma, el gorila) o bien variables (el perro / la perra, el león / la leona, el palomo / la paloma), pero con la particularidad de que el término genérico de estos últimos no necesariamente se identifica con el masculino, como sucede con los variables de persona, pues la designación de la especie animal también puede realizarse mediante el nombre femenino: las palomas incluye a palomas y palomos, lo mismo que las jirafas, las panteras, las ballenas, las ardillas, etc. A estas dos clases aún habría que añadir el grupo reducido de animales que recibe un nombre diferente para cada sexo sin relación etimológica, como caballo y yegua, toro y vaca, carnero y oveja. Estos nombres suelen denominarse heterónimos en las gramáticas. La clase de los heterónimos también incluye algunos nombres personales, como padre y madre, papá y mamá, padrino y madrina. Todos estos, sean de animales o de persona, comparten dos propiedades por las que se 
distinguen de las otras clases. Por un lado, frente a los epicenos, no aceptan la aposición de los términos hiperónimos macho / hembra, varón / mujer, masculino / femenino: *una oveja hembra, *un caballo macho, *un padre varón, construcciones que resultarían usuales en el caso de la pantera macho, la ballena hembra, las autoridades femeninas. Por otro lado, como los variables, pero a diferencia de los ortónimos -que se presentarán a continuación-, disponen de un término no marcado para la referencia a ambos sexos que no necesariamente se identifica con el masculino, salvo en los nombres personales: los padres suele incluir al padre y a la madre, pero un rebaño de ovejas también puede contar con carneros y la cría de caballos puede referirse indistintamente a los caballos y a las yeguas.

En resumen, las tres clases de nombres, de animales, personas y cosas, admiten la variación de género, pero el masculino genérico es una propiedad exclusiva de los nombres que designan clases de personas. Es más, sin un masculino genérico disponible, no se forma un nombre de género variable, aunque las terminaciones coincidan con las usuales del masculino y femenino. Así, por ejemplo, jequesa no es el femenino de jeque, 'soberano de un territorio', sino que designa la esposa de este. A diferencia de alcalde y alcaldesa, jeque y jequesa no son sustantivos variables. De ahí que jeques no se use como masculino genérico, pues solo incluye a varones.

Las pruebas gramaticales del comportamiento del masculino como término no marcado se refieren, de una parte, a la selección de formas adjetivas que no se distinguen morfológicamente del masculino en los casos de concordancia con elementos neutros, como Aquello fue maravilloso; Esto no es del todo cierto. El grupo neutro de lo más, de carácter superlativo, concuerda normalmente con adjetivos en masculino si se predica la propiedad a un conjunto de diferentes entidades: Las ofertas son de lo más variado. Con oraciones sustantivas, se observa igualmente la presencia sistemática del masculino: Está claro que se despistó; Es necesario mantener la calma. Las sustantivaciones del infinitivo y de cualquier otro elemento se construyen también con artículos o adjetivos en masculino:

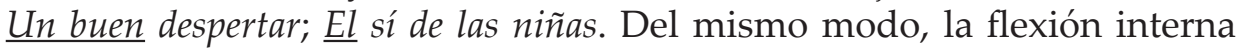
de los compuestos se presenta con una terminación que no se distingue formalmente de la del masculino: Una chica sordomuda; Las relaciones hispanoamericanas; El agua mineromedicinal; Las propiedades fisicoquímicas; Las transmisiones paternofiliales. Los adjetivos, en fin, no presentan formas distintas del masculino singular en la función adverbial, como en trabajar duro, hablar claro, volar bajo, respirar hondo, jugar limpio. 


\section{Clases de nombres personales}

En los nombres de persona y, en general, de individuos animados, por otra parte, el uso del masculino genérico no es sino la consecuencia del carácter no marcado de esta desinencia flexiva. Tal propiedad del género masculino está, como señala la Gramática académica (NGLE, $2009, \S 2.2 . k$ ), tan fuertemente arraigada en el sistema gramatical del español que convendría, por nuestra parte, añadir que el factor gramatical que impide la interpretación genérica del masculino depende de la clase del nombre de persona de que se trate. Pues bien, además de los heterónimos, dos de las cinco clases que se distinguen en el conjunto de los nombres referidos a personas, los nombres variables (compañero, ra; niño, ña) y los comunes (el estudiante, la estudiante; el periodista, la periodista) incluyen en la flexión del masculino la referencia a individuos de ambos sexos siempre que el enunciado no contenga algún atributo o predicado exclusivo de los varones (como sería, por ejemplo, en Dos españoles alcanzaron el cardenalato; Los burócratas de la curia romana). Sin este tipo de especificaciones contextuales marcadas para las referencias masculinas, los nombres variables y comunes en masculino se interpretan genéricamente: Dos españoles alcanzaron la presidencia; Los burócratas de la administración europea. Este carácter no marcado del masculino se manifiesta igualmente en la concordancia con nombres de persona de distinto género coordinados, como en El padre y la madre estaban separados; El rey y la reina no viajaron juntos; Juan y María son muy parecidos; El testigo y la testigo permanecieron callados. Con estos mismos nombres de persona, pero solo en masculino por ser el no marcado, son frecuentes los enunciados en los que se especifica el sexo del individuo mencionado: Todavía no saben el sexo de su hijo; Los gemelos son niño y niña; Los testigos fueron un hombre y una mujer; Su primer nieto ha sido niña. Las variantes en femenino, por el contrario, resultarían anómalas ( ${ }^{*}$ Todavía no saben el sexo de su hija; ${ }^{*}$ Las gemelas son niño y niña).

Pero no todas las clases de nombres de persona en masculino presentan este carácter genérico. En efecto, otra clase de nombres de persona, que hemos denominado ortónimos, carece de esta posible interpretación genérica porque se trata de nombres invariables, pero con la particularidad de que los de género masculino designan sistemáticamente varones y los de género femenino solo se refieren a mujeres. Está claro que los ortónimos masculinos, en singular o en plural, carecen de función genérica independientemente de cualquier tipo de contexto, como se observa en los ejemplos siguientes: 
Todos los párrocos de la provincia estaban presentes.

Había invitado a sus yernos.

Eran cuatro jóvenes seminaristas.

Los maridos acababan de llegar.

Los caballeros vestían de rigurosa etiqueta.

La obra será interpretada por dos tenores y tres barítonos.

Muchos cronistas eran clérigos.

Algunos nombres de profesiones se codifican recientemente como variables pues han desarrollado en la actualidad una forma masculina a partir de una femenina previa, que designaba ocupaciones propias y exclusivas de mujeres. Estos ortónimos femeninos, como azafata, enfermera, comadrona, admiten ahora la flexión en masculino, azafato, enfermero, comadrón, pero con la particularidad de que rara vez se emplean estos masculinos como genéricos (Borrego Nieto, 2016: 255). Se comportan, pues, como nuevos ortónimos masculinos, del mismo modo que modisto.

Los heterónimos forman otra clase invariable de nombres de persona. En general, las gramáticas suelen ilustrar la heteronimia tanto con sustantivos como padre y madre como con otras parejas del tipo yerno y nuera, marido y mujer, además de algunos nombres de animales. Los nombres heterónimos presentan, en efecto, una raíz diferente para distinguir el masculino del femenino. Pero, aquí se observa que tal característica no parece ser suficiente en la definición de esta clase de nombres, pues los heterónimos, como padre y madre, papá y mamá, padrino y madrina se comportan como los nombres variables y comunes en el sentido de que el de género masculino funciona como término no marcado en las mismas condiciones contextuales que estos nombres flexivos: en Los padres de Ana se fueron de vacaciones, se entiende normalmente que el padre y la madre de Ana están de viaje; en Mis padrinos me regalaron una bicicleta, se supone que el padrino y la madrina hicieron el regalo. En cambio, los ortónimos como yerno y nuera, marido y mujer, caballero y dama, etc., no disponen de un masculino genérico: los yernos no incluye a las nueras; en los caballeros solo se habla de varones.

Por el contrario, la quinta y última clase de nombres que designa individuos animados (personas y animales), los llamados epicenos, también invariables, no aportan información semántica sobre el sexo del referente. Se caracterizan por desvincularse de esta correspondencia entre el género gramatical y el sexo que solamente presentan los ortónimos, de modo que el valor genérico se alcanza tanto en los epicenos masculinos como en los epicenos femeninos. A continuación se ejemplifica esta propiedad:

La policía busca a los sujetos del robo, posiblemente un hombre y una mujer.

El ser humano, hombre o mujer, puede resultar despiadado. 
Las autoridades municipales estaban representadas por la alcaldesa y los concejales. Las personas adultas, hombres y mujeres, no se comportan así.

Nótese que las expresiones formadas por la coordinación de los dos géneros de una misma palabra flexiva para expresar la referencia a personas de ambos sexos carecen de justificación (excepto en los vocativos o saludos de cortesía, como en Buenas noches, señoras y señores, pues a estos no les afectan las relaciones de concordancia, como recuerda Martínez, 2008) siempre que sea compartido el contenido de la información, sin establecer diferencias entre los referentes de uno y otro sexo, como en los siguientes ejemplos:

(?) El éxito de la democracia dependerá de todos nosotros y todas nosotras.

(?) Juntos y juntas hemos hecho un gran equipo.

(?) Los jueces y las juezas les acusan de violencia callejera.

(?) Los decanos y las decanas de las Facultades firmaron el manifiesto.

(?) Los bomberos han evacuado a treinta vecinos y vecinas de la zona.

Este tipo deenunciados, generalmentelimitado hoy al discurso político, sindical, escolar, etc., representa el retroceso a una etapa del español en la que la ortonimia predominaba sobre los nombres variables y comunes $(\mathrm{el}$ torero, por ejemplo, solo podía referirse a un varón). El idioma reflejaba las diferencias sociales entre hombres y mujeres, de modo que los nombres de persona presentaban acepciones diferentes según el género, pues muy frecuentemente el masculino significaba un oficio, cargo o profesión, pero el femenino no designaba un referente femenino de igual significado, sino que, como indican todavía los diccionarios, denotaba 'la esposa de' quien se tratara. En esta situación, el masculino carecía de función genérica y es natural que con la reiteración de nombres ortónimos de uno y otro género se tratara de expresar la diferencia de acepciones en cada caso. Una selección de textos antiguos muestra algunos de estos usos (CORDE):

Las intrigas [...] que se traen [...] el alcalde y la alcaldesa, el maestro y la maestra, el secretario y su novia, [...] el cura y su ama.

Vinieron el ventero y la ventera muy paso entre paso, alumbrando ella con un cabo de vela; el marido comenzó a desviar con mucho silencio un gran montón de estiércol.

Una mujer tuvo una cuestión con la esposa de un carabinero, insultándose mutuamente. Entonces la carabinera le dio un mordisco.

La señora del alcalde era una dama bonachona, sin otra flaqueza que suponerse muy relacionada en la corte [...]. La alcaldesa hablaba por los codos.

Una fiesta sonada [...] convidando a todo el Madrid explotable, desde la presidenta consorte del comité carlista, hasta la ministra cesante, esposa dignísima del excelentísimo señor don Juan Antonio Martínez. 


\section{Los nombres comunes en cuanto al género}

Muchos nombres apelativos que designan clases de personas, los llamados comunes en cuanto al género, son invariables, de modo que la referencia a uno u otro sexo, si es el caso, no se obtiene mediante un cambio de desinencia, como en los nombres variables alumno, alumna; presidente, presidenta, sino por la concordancia con determinantes $\mathrm{y}$ adjuntos de uno u otro género, como en el piloto español, la piloto española. Sin embargo, no sería acertado considerar hoy que estos nombres comunes en cuanto al género son defectivos, irregulares o anómalos por presentarse invariables a la flexión de género para expresar la referencia a individuos de uno $u$ otro sexo, como a veces se ha sugerido. Conviene recordar, en primer lugar, que este tipo de nombres únicamente designa clases de personas según sus profesiones, oficios, cargos, edades, etc., como acróbata, artista, astronauta, cómplice, consorte, cónyuge, forense, joven, miembro, protagonista, residente, testigo, sicópata, etc. No hay nombres comunes en cuanto al género para denominar clases de animales ni clases de cosas (los ambiguos, como el mar, la mar, son sinónimos). Pero, por otro lado, la condición de defectivos o anómalos para los nombres comunes en cuanto al género, e incluso para los epicenos (persona), obligaría a defender que la regularidad se presenta solo en los nombres variables $\mathrm{y}$, más concretamente, en el paradigma flexivo que se ajusta a la alternancia de $-o,-e,-\varnothing$ para el masculino y -a para el femenino (hijo / hija, jefe / jefa, lector / lectora) y que además esta clase de nombres variables con moción de género representa el paradigma dominante y más productivo en el español actual. Sin embargo, estas desinencias no son las únicas para distinguir el masculino del femenino (barón / baronesa, rey / reina, zar / zarina). Tampoco la terminación -a es la única posible para la formación de los variables femeninos: institutriz, emperatriz, actriz.

Pero habría que añadir además que los nombres comunes en cuanto al género representan en el español actual un paradigma muy productivo y de una importante expansión durante el último siglo. Al crecimiento de los nombres comunes en cuanto al género han contribuido varios factores, pero el principal consiste en que la condición sintáctica del género en español, basada en las relaciones de concordancia, permite prescindir de las desinencias, como se comprueba igualmente con los pronombres referidos a personas que se comportan como comunes en cuanto al género: yo, tú, usted, alguien, quién, etc. (tú mismo tú misma). Se pueden entender así algunos cambios y resultados como los siguientes: 
1) el desuso de algunas formaciones en -esa, -isa, empleadas hoy como nombres comunes: el / la cónsul, el / la poeta, etc., en lugar de la consulesa, la poetisa, etc.; 2) la competencia entre los variables y los comunes que se registra en la actualidad en muchos nombres de oficios y profesiones: $\mathrm{el}$ aprendiz / la aprendiza o la aprendiz, el capataz / la capataza o la capataz, el fiscal / la fiscala o la fiscal, el juez / la jueza o la juez, etc.; 3) el uso generalizado como nombres comunes de los préstamos: el / la chef, el / la chófer, el / la fan, el / la jipi (o hippy), el / la líder, el / la maître, el / la paparazzi, el / la piloto, el / la pívot, el / la sumiller, etc.; 4) los acortamientos de nombres personales también se usan como comunes: profe, progre, compa, facha, guiri, machaca, nazi, narco, otorrino, etc.; 5) también se usan mayoritariamente como comunes los nombres compuestos: el / la aguafiestas, el / la guardabosques, el / la limpiabotas, el / la picapleitos, el / la portavoz, el / la sacamuelas, etc.; 6) las acepciones referidas a clases de personas en el caso de lemas polisémicos que cuentan con otras acepciones de nombres de cosas también suelen usarse como comunes: el / la botones, el / la guía, el / la miembro, el / la testigo, etc.; 7) la sustantivación de adjetivos de una sola terminación: el / la auxiliar, el / la forense, el / la parricida, etc.; 8) la reclasificación de series léxicas completas, como los nombres de los grados militares, que no contaban con apelativos femeninos, como el / la soldado, el / la cabo, el / la capitán, el / la teniente, etc. Estas nuevas clasificaciones contribuyen a explicar la fuerte expansión de los nombres comunes en cuanto al género en el español actual.

\section{Bibliografía}

AMBADIANG, T. (1994). La morfología flexiva. Madrid: Taurus.

BORREGO NIETO, J. (dir.) (2016). Cocodrilos en el diccionario. Barcelona: Instituto Cervantes.

CORDE - Real Academia Española, Banco de datos (CORDE). Corpus diacrónico del español [en línea] <http://www.rae.es>, fecha de consulta: 17 de enero de 2017.

LLITERAS, M. (2008). «Del género derivativo al género flexivo», en M. MAQUIEIRA y M. D. MARTÍNEZ GAVILÁN (eds.), Gramma-Temas, vol. 3: España y Portugal en la tradición gramatical. León: Centro de Estudios Metodológicos e Interdisciplinares / Universidad de León, 125-148.

LLITERAS, M. (2010). «Problemas de codificación de los nombres personales en los siglos XIX y XX», en C. ASSUNÇÃO, G. FERNANDES y M. LOUREIRO (eds.), Ideias Linguísticas na Península Ibérica. Münster: Nodus Publikationen, 513-523. 
LLITERAS, M. (2014). «Los nombres ortónimos en tiempos de la fundación de la Real Academia Española», en M. L. CALERO et al. (eds.), Métodos y resultados actuales en Historiografía de la Lingüistica. Münster: Nodus Publikationen, 434-444.

MARTÍNEZ, J. A. (2008). El lenguaje de género y el género lingüístico. Oviedo: Universidad de Oviedo.

NGLE - Real Academia Española y Asociación de Academias de la Lengua Española (2009). Nueva gramática de la lengua española. Madrid: Espasa Libros.

PICALLO, M. C. (2016). «Género y número», en J. GUTIÉRREZ-REXACH (ed.), Enciclopedia de Lingüística Hispánica. London / New York: Routledge, 630-639. 